\title{
PyMelt: An extensible Python engine for mantle melting calculations
}

\author{
Simon Matthews ${ }^{* \dagger}$ Kevin Wong, ${ }^{\ddagger}$ Matthew Gleeson ${ }^{\S}$
}

\begin{abstract}
Modelling the melting of Earth's mantle is crucial for understanding the distribution of volcanic activity on Earth and for testing models of mantle convection and mantle lithological heterogeneity. PyMelt is a new open-source Python library for calculating the melting behaviour of multi-lithology mantle and can be used to predict a number of geophysical and petrological observations, including melt productivity, spreading centre crustal thickness, lava trace element concentrations, and olivine crystallisation temperatures. The library is designed to be easily extensible, allowing melting models to be added, different methods for calculating lava chemistry to be applied, and new melting dynamics and properties to be incorporated.
\end{abstract}

\section{INTRODUCTION}

Models for melting the Earth's mantle have been used widely in studies of Earth's convecting mantle, often to provide quantitative constraints on mantle temperature variability [e.g., Ball et al., 2021], mantle compositional variability [e.g., Brown and Lesher, 2014; Gleeson et al., 2021], or as a means of estimating primary melt compositions [e.g., Jennings et al., 2016]. These melting models calculate mantle melting behaviour either by minimising thermodynamic potentials at each calculation step [e.g., Smith and Asimow, 2005], or by using expressions parameterised directly from melting experiments [e.g., Lambart et al., 2016]. The parameterised approach is particularly useful for calculations requiring many runs of a melting model, for example when inverting for mantle properties from geochemical or geophysical observations [e.g., McKenzie and O’Nions, 1991; Matthews et al., 2021].

PyMelt is an open-source extensible Python library that employs the parameterised approach, providing a powerful and flexible tool for calculating the melting behaviour of lithologically heterogeneous mantle. The library incorporates existing

\footnotetext{
${ }^{*}$ Institute of Earth Sciences, University of Iceland, Reykjavík, Iceland

${ }^{\dagger}$ Corresponding author: simonm@hi.is, @simonwmat thews UK

‡School of Earth and Environment, University of Leeds, Leeds,

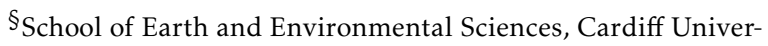
sity, Cardiff, UK
}

melting parameterisations (Section 3), in addition to methods for estimating igneous crustal thickness, magmatic productivity, lava trace element concentrations, and olivine crystallisation temperatures. The library is designed to be extensible: new models can be added to the library very simply, in addition to new functions for predicting geochemical and geophysical observations.

A number of software tools are available that can perform similar, though more limited, calculations, including INVMEL [McKenzie and O'Nions, 1991], REEBOX-PRO [Brown and Lesher, 2016], MELTPX [Lambart et al., 2016], and BDD21 [Ball et al., 2022]. While these packages have been used extensively in studies of mantle melting, pyMelt offers a number of advantages. PyMelt is the only package that is simultaneously open-source, incorporates mantle lithological heterogeneity, and can be easily integrated with other Python libraries (e.g., Monte-Carlo inversion tools). Open-source software is an essential part of open, transparent, and reproducible science, and it provides the basis for the development of more advanced codes and integration with other libraries. The importance of modelling the effects of lithological heterogeneity on mantle melting behaviour for accurately predicting magmatic productivity, melt compositions, and magmatic teperatures is becoming increasingly clear [Phipps Morgan, 2001; Pertermann and Hirschmann, 2003; Shorttle et al., 2014; Matthews et al., 2021]. PyMelt therefore occupies an important niche in mantle melting calculations 
and will provide the basis for solving new problems related to melt generation and magmatism for many years to come.

In this manuscript we review the main features of pyMelt, the library structure, the underlying mathematical formulation, and the computational approaches it takes. Users are directed to the pyMelt documentation (pyMelt.readthedocs.io) for a comprehensive guide to using the library, and to the interactive tutorials available on myBinder. The pyMelt repository is hosted on GitHub (github.com/simonwmatthews/pyMelt), where the code can be obtained, bugs reported, new features requested, and new contributions made. PyMelt can also be installed directly using the pip package manager.

\section{PyMelt structure}

Figure 1 summarises the modular structure of the pyMelt library and its workflow. The melting behaviours of individual lithologies (e.g., lherzolite or pyroxenite) are contained with lithology classes (Section 3), which can be combined in specified mass fractions $\phi$ to form a Mantle class (Section 4). Any lithology class in pyMelt can be converted to a hydrousLithology class where the effect of water on its solidus and melt productivity is estimated (Section 5). An adiabatic decompression calculation can then be performed on a Mantle class instance at a specified mantle potential temperature $T_{p}$ (the temperature a parcel of mantle would have following decompression to 0 bar while undergoing no chemical changes) using its adiabaticMelt method (Section 4) which returns a meltingColumn instance. If desired, the trace element concentrations in these melts can be calculated by calling the calculateChemistry method of the meltingColumn class (Section 6). To calculate geological setting specific properties, such as crustal thickness $t_{c}$ at a mid-ocean ridge, a geoSetting class can be created using the meltingColumn class instance (Section 7). The results can then be extracted from the geoSetting class instance and plotted, used in further calculations, or saved (see the tutorial notebooks).

\section{Mantle lithologies}

The experiments that are used to parameterise melting models are performed on particular bulk compositions, or lithologies. This means that each melting model, unless parameterised also for bulk composition [e.g., Lambart et al., 2016], represents a particular lithology, with its own melting behaviour. At a minimum, the lithology class has methods defined for the solidus and liquidus temperatures as a function of pressure $P$ (TSolidus and TLiquidus), and for the melt fraction $F$ as a function of $P$ and

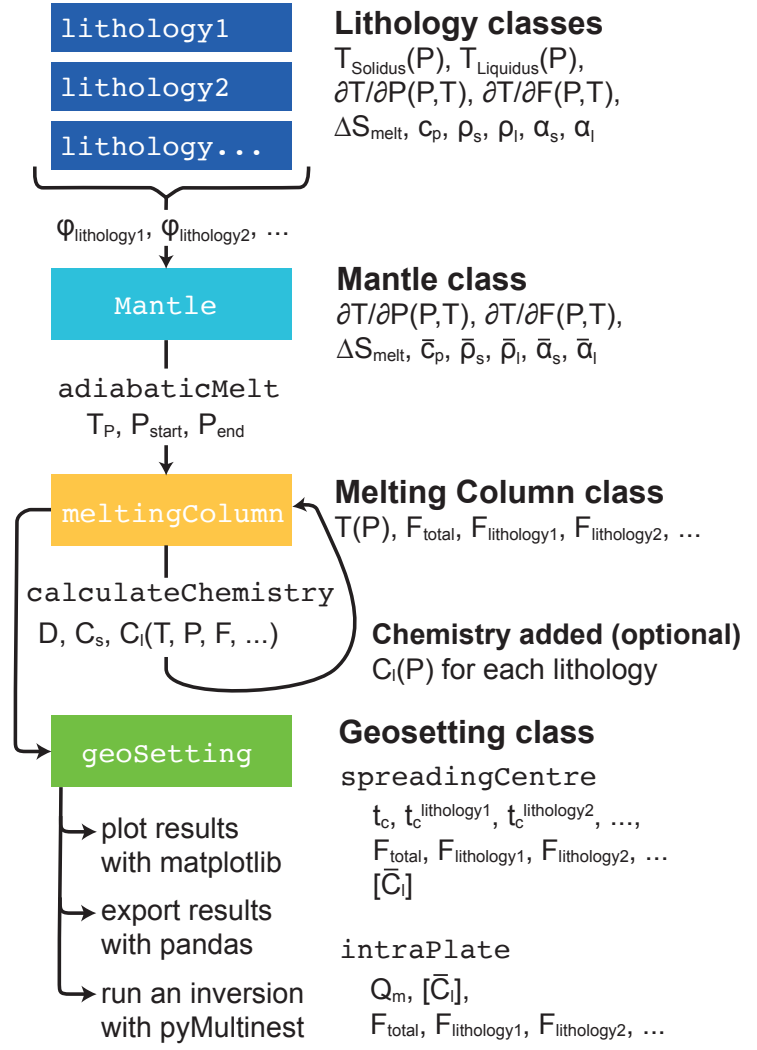

Figure 1: Summary of the structure of pyMelt. Each box represents an instance of a pyMelt class, either created directly by the user (with variables as specified on the arrows) or returned by a method call. The properties and methods of each class are shown on the right hand side (symbols as defined in the text).

temperature $T(\mathrm{~F})$. The models contained within pyMelt already have methods for $\left(\frac{\partial T}{\partial F}\right)_{P}(\mathrm{dTdF})$ and $\left(\frac{\partial T}{\partial P}\right)_{F}(\mathrm{dTdP})$, but pyMelt can also calculate their values numerically from the TSolidus, TLiquidus, and $\mathrm{F}$ methods. Additionally, each lithology has values associated with it for the density of the solid lithology $\rho_{s}$ and its melt $\rho_{l}$, their thermal expansivities $\alpha_{s}$ and $\alpha_{l}$, the heat capacity $C_{p}$, and the entropy change on melting $\Delta S$.

Table 1 lists the melting models for which lithology classes are defined in pyMelt. The pyroxenitic lithologies are categorised as having a relative excess or deficit in silica, with the silica-excess models in pyMelt representing a mid-ocean ridge basalt like composition, and the silica-deficient pyroxenites representing a mixture of basalt and lherzolite.

\section{Mantle melting}

Before melting calculations can be performed, the lithology class instances must be assembled in a Mantle class, with their relative mass fractions spec- 
Table 1: The pure lithology melting models built into pyMelt. See Lambart et al. [2016] for a description of the pyroxenite classification.

\begin{tabular}{|c|c|c|}
\hline Reference & Class name & Lithology type \\
\hline McKenzie and Bickle [1988] & mckenzie.lherzolite & Lherzolite \\
\hline Pertermann and Hirschmann [2003] & pertermann.g2 & Pyroxenite (silica-excess) \\
\hline Katz et al. [2003] & katz.lherzolite & Lherzolite \\
\hline Shorttle et al. [2014] & shorttle.kg1 & Pyroxenite (silica-deficient) \\
\hline \multirow[t]{3}{*}{ Matthews et al. [2021] } & $\begin{array}{l}\text { mat thews.klb1 } \\
\text { mats }\end{array}$ & Lherzolite \\
\hline & matthews.kg1 & Pyroxenite (silica-deficient) \\
\hline & matthews.eclogite & Pyroxenite (silica-excess) \\
\hline \multirow[t]{3}{*}{ Ball et al. [2022] } & ball.depleted & Lherzolite \\
\hline & ball.primitive & Lherzolite \\
\hline & ball.mixed & Lherzolite \\
\hline
\end{tabular}

ified. pyMelt does not limit the number of lithologies that can be assembled in a Mantle class, though in most situations one each of a lherzolite, pyroxenite, and harzburgite lithology are sufficient. The Mant le class replicates many of the properties of the lithology class (Figure 1), with methods returning either the mass-weighted average properties, or an array with each lithology's value. Implicit in our treatment of the lithology objects, and our application of the melting formulation by Phipps Morgan [2001], is an assumption of complete thermal equilibrium but complete chemical disequilibrium between lithologies.

Adiabatic decompression melting calculations are performed by the adiabaticMelt method of the Mantle class, requiring only that a value for $T_{p}$ is specified. By default the calculation will begin at the solidus and end at $0.01 \mathrm{GPa}$ with a pressure decrement of $0.004 \mathrm{GPa}$ at each decompression step; though all of these parameters can be modified.

The calculation proceeds by simultaneously integrating $\frac{d F}{d P}$ for each lithology $i$, and $\frac{d T}{d P}$ for the melting assemblage, to obtain the melt fractions $\left(F_{i}\right)$ of each lithology and the mantle temperature $(T)$ at each step. The value of $\frac{d F}{d P}$ is determined for each melting lithology using Eq. 29 of Phipps Morgan [2001] (the mass-weighted average values of $c_{p}, \alpha$, and density $\rho$ are indicated with a bar):

$$
\frac{d F_{i}}{d P}=-\frac{\frac{\overline{c_{p}}}{T} \frac{\partial T_{i}}{\partial P}-\frac{\bar{\alpha}}{\bar{\rho}} \sum_{n \neq i}\left[\phi_{n} \Delta S_{n}^{m} \frac{\frac{\partial T_{i}}{\partial P}-\frac{\partial T_{n}}{\partial P}}{\frac{\partial T_{n}}{\partial F_{n}}}\right]}{\phi_{i} \Delta S_{i}^{m}+\sum_{n \neq i}\left[\phi_{n} \Delta S_{n}^{m} \frac{\frac{\partial T_{i}}{\partial F_{i}}}{\left.\frac{\partial T_{n}}{\partial F_{n}}\right]+\frac{\bar{c}_{p}}{T}} \frac{\partial T_{i}}{\partial F_{i}}\right.}
$$

The value of $\frac{d T}{d P}$ is then obtained from Eq. 28 of Phipps Morgan [2001] using the values for one lithology $j$ (arbitrarily, the one with the most negative $\frac{d F}{d P}$ in pyMelt):

$$
\frac{d T}{d P}=\frac{d T_{j}}{d P}+\frac{d T_{j}}{d F_{j}} \frac{d F_{j}}{d P}
$$

The integration is performed using a fourth-order Runge-Kutta routine. The results of the melting calculation are returned as a meltingColumn class, which records the melt fractions of each lithology, in addition to the aggregate melt fraction and the temperature at each pressure step.

When the calculation is started at a specified pressure, high mantle $T_{p}$ may mean the mantle has already exceeded its solidus. In this case an interval of isobaric melting will occur before decompression starts, such that entropy is conserved. The computational method for this isobaric step is described in the supporting information of Matthews et al. [2021]. Decompression melting then proceeds, as described above.

Since the Phipps Morgan [2001] melting formulation assumes batch melting (whereby the melt is not separated from the solid residue), when performing calculations where one lithology is more fusible than another lithology $j$, the heat extracted by melting of the more fusible lithology can cause $d F_{j} / d P>0$ (i.e., refreezing) in the other lithology. By default pyMelt will prevent freezing from occurring by setting $d F_{j} / d P=0$, thereby more closely representing continual melt extraction. This is set as the default behaviour because the chemistry module requires monotonically increasing melt fractions.

\section{Hydrous melting}

Any lithology in pyMelt can be turned into a hydrous lithology using the hydrousLithology class. To approximate the effect of hydrous melting a similar formulation to that developed by Katz et al. [2003] is used. In this formulation the solidus temperature is depressed according to their Eq. 16:

$$
T_{\text {solidus }}^{\text {hydrous }}=T_{\text {solidus }}-K X_{\mathrm{H}_{2} \mathrm{O}}^{\gamma}
$$

where $K$ and $\gamma$ are constants and $X_{\mathrm{H}_{2} \mathrm{O}}$ is the water concentration in the melt in wt\%. This differs slightly from the equations developed by Katz et al. [2003] as they did not apply the $-K X_{\mathrm{H}_{2} \mathrm{O}}^{\gamma}$ term to every instance of $T_{\text {solidus }}$ in their expressions (e.g., their Eq. 19). The amount of water that can be 
dissolved in magmas is limited, but increases with pressure. Katz et al. [2003] model this effect using (their Eq. 17):

$$
X_{\mathrm{H}_{2} \mathrm{O}}^{\mathrm{sat}}=\chi_{1} P^{\lambda}+\chi_{2} P, \quad 0<\lambda<1
$$

which is also implemented in pyMelt.

The concentration of $\mathrm{H}_{2} \mathrm{O}$ present in the melt decreases as melting proceeds, owing to $\mathrm{H}_{2} \mathrm{O}$ partitioning favourably into the melt and being continually diluted by new additions of magma. Katz et al. [2003] modelled this change by using the batch melting equation:

$$
X_{\mathrm{H}_{2} \mathrm{O}}=\frac{X_{\mathrm{H}_{2} \mathrm{O}}^{\text {bulk }}}{D_{\mathrm{H}_{2} \mathrm{O}}+F\left(1-D_{\mathrm{H}_{2} \mathrm{O}}\right)}
$$

where $D_{\mathrm{H}_{2} \mathrm{O}}$ is the partition coefficient during melting. We extend this formulation to consider also the removal of $\mathrm{H}_{2} \mathrm{O}$ from the system by near-fractional melting, as modelled previously by Asimow et al. [2003]; however, since the melting models in pyMelt are implicitly expressions of batch (or equilibrium) melting, modelling the effect of $\mathrm{H}_{2} \mathrm{O}$ extraction by fractional melting cannot be done entirely selfconsistently. The effect is approximated in pyMelt by replacing Eq. 5 with an expression for nearfractional melting:

$$
X_{\mathrm{H}_{2} \mathrm{O}}=\frac{X_{\mathrm{H}_{2} \mathrm{O}}^{\mathrm{bulk}}}{(1-\phi) D+\phi}(1-F)^{\frac{(1-\phi)(1-D)}{(1-\phi) D}}
$$

where $\phi$ is the porosity during melting.

When a hydrousLithology class is created, the supporting methods from the original lithology class are copied, along with the TLiquidus method (which is not changed by the hydrous melting extension). A new method for TSolidus is defined, which applies Eq. 3 to the original TSolidus method. Since the value of $F$ depends on $X_{\mathrm{H}_{2} \mathrm{O}}$, which itself depends on $F$, a new $F$ method is created, which solves the equation:

$$
F_{\text {calc }}\left(P, T, F_{\text {guess }}\right)-F_{\text {guess }}=0
$$

where $F_{\text {calc }}()$ is the original $F$ method (which will provide the hydrous melt fraction as it calls the modified TSolidus method) and $F_{\text {guess }}$ is the value changed by the root finding method. pyMelt uses the brentq algorithm implemented in the SciPy.optimize.root_scalar method [Virtanen et al., 2020]. New methods for $\left(\frac{\partial T}{\partial F}\right)_{P}$ and $\left(\frac{\partial T}{\partial P}\right)_{F}$ are created, which calculate the values numerically using SciPy.misc.differentiate, alongside SciPy.optimize.root_scalar to find the T-P curve at constant $F$.

The default values for $K, \gamma, \chi_{1}, \chi_{2}, \lambda$, and $D_{\mathrm{H}_{2} \mathrm{O}}$ are taken from Katz et al. [2003] who calibrated them for hydrous-lherzolite melting. While pyMelt provides the opportunity to model hydrouspyroxenite melting in the same way, the user must choose appropriate constant values for pyroxenite.

\section{Trace elements}

Following the creation of a meltingColumn class by the mantle.adiabaticMelt method, the pyMelt chemistry module can be used to calculate the trace element contents of the melts $C_{i, l}$ (Figure 1). The calculation is performed by the meltingColumn.calculateChemistry method and requires the concentration of each trace element in each lithology $C_{i, s}$, in addition to the parameters required by the chemical model (e.g., the partition coefficients $D_{i}$ ). There are four built in chemical models: batch melting, near-fractional melting (instantaneous and accumulated melts), and the INVMEL forward model [McKenzie and O'Nions, 1991]. For the batch and near-fractional melting models the partition coefficient can either be a constant, or a user defined function of $F, P$, and $T$.

Each element (in each lithology) to be included in the calculation is defined as a species class instance that contains its solid concentration $c_{0}$ and a composition method for calculating the melt composition as a function of $F$ (and possibly $P$ and $T$ ). Defining each element separately permits the incorporation of more complex partitioning behaviour for some elements alongside simpler models for other elements. Generally, users will be unaware of the species class: the meltingColumn. calculateChemistry method can assemble them automatically.

The INVMEL model incorporates the effects of phase changes and phase exhaustion on the partioning of trace elements, in particular the effects of garnet- and clinopyroxene-present melting, but requires many more parameters to be defined. The partition coefficients used by default are those compiled by Gibson and Geist [2010], and other parameters are set to the values used by Ball et al. [2021].

For convenience, the chemistry module has a number of estimates for partition coefficients and mantle trace element concentrations built in (see the documentation for more details).

\section{Geological Settings}

In many cases the information provided by the meltingColumn object is sufficient; in other cases a user may be interested in derived properties for a particular geological setting, aggregate melt compositions for example. The pyMelt geoSetting classes (spreadingCentre and intraPlate) provide this facility, taking a meltingColumn instance as an input (Figure 1).

When calculating aggregate properties of the melting region we must consider how each melt in the melting column should be weighted to account for mantle flow. For example, active upwelling in a mantle plume causes more mantle ma- 
terial to pass through the melting region at its base [Maclennan et al., 2001], meaning deeper melts should have a greater weighting in plume models. User defined weighting functions may be specified for each calculation, but how they are implemented varies between geoSetting classes. An example weighting function built into pyMelt (geosettings.weighting_expdecay) has the form:

$$
w(P)=\mu \exp \left(-\frac{1}{\lambda} \frac{P_{\max }-P}{P_{\max }-P_{\min }}\right)
$$

where $\lambda$ and $\mu$ are constants, and $P_{\max }$ and $P_{\min }$ are the maximum and minimum pressures from which melts are formed at the geological setting.

\subsection{Spreading centres}

When an instance of the spreadingCentre class is initialised, the crustal thickness will be calculated, assuming passive corner-flow mantle upwelling [Plank and Langmuir, 1992]. To account for the triangular melting region, the total melt fraction is integrated over the melting column, until the pressure exerted by the crust (calculated by stepwise integration with the trapezium rule) is equal to the pressure of the melting step:

$$
t_{\mathrm{c}}=\frac{1}{g \rho_{\mathrm{c}}} \int_{P_{\text {start }}}^{P_{\text {crust }}}(1+w) \frac{\sum \phi_{i} F_{i}}{1-\sum \phi_{i} F_{i}} d P
$$

where $g$ is the acceleration due to gravity on Earth, and $\rho_{\mathrm{c}}$ is the density of the crust. The term $w$ is the optional user-defined weighting function, which takes $w=0$ by default. Using the form $1+w$ allows separation of the passive upwelling component and the active upwelling component. The $\left(1-\sum \phi_{i} F_{i}\right)$ term in the denominator accounts for compaction, i.e., mantle material will continuously replace the volume lost due to melt extraction [White et al., 1992]. The contributions of each lithology to the aggregate crust is calculated similarly:

$$
t_{\mathrm{c}}^{n}=\frac{1}{g \rho_{\mathrm{c}}} \int_{P_{\text {start }}}^{P_{\text {crust }}}(1+w) \frac{\phi_{n} F_{n}}{1-\sum \phi_{i} F_{i}} d P
$$

When modelling continental rifts, the pressure exerted by the lithosphere can be imposed and the thickness of igneous crust calculated.

If the meltingColumn class used to generate the spreadingCentre class has chemistry, upon initialisation of the spreadingCentre class, the composition of the homogenised melt is calculated. Similarly to the crustal thickness calculations, homogenisation of chemistry takes into account the triangular melting region, compaction and any additional weighting function. The equation is modified from McKenzie and O'Nions [1991]:

$$
\bar{C}=\frac{\int_{P_{\text {start }}}^{P_{\text {end }}}(1+w) \frac{\sum \phi_{i} F_{i} C_{l, i}}{1-\sum \phi_{i} F_{i}} d P}{\int_{P_{\text {start }}}^{P_{\text {end }}}(1+w) \frac{\sum \phi_{i} F_{i}}{1-\sum \phi_{i} F_{i}} d P}
$$

and is evaluated using the trapezium rule. If the calculated melt compositions represent instantaneous and not batch melts, each column is first homogenised, with each melt weighted according to its lithology fraction and melt fraction, as above. The weighting function $w(P)$ is not applied in this step.

The crystallisation temperature of melts extracted from the top and base of the melting region can be calculated, using the method described by Matthews et al. [2016]. The olivine saturation temperature at the pressure of magma storage is found using the pressure dependence of the olivine saturation surface [39.16 $\mathrm{K} \mathrm{GPa}^{-1}$, Putirka, 2008]. This method is available also in the intraPlate geoSetting class.

\subsection{Intra-Plate settings}

To initialise an instance of the intraPlate geoSetting class, the pressure at the base of the lithosphere must be provided. The calculation results stored in the meltingColumn will be truncated at that pressure. If the relative density of the mantle is provided $\left(\Delta \rho=\rho_{\text {ambient-mantle }}-\rho_{\text {plume-mantle }}\right)$, the melt flux $Q_{m}$ is calculated during initialisation using the equation:

$$
Q_{m}=\frac{\pi}{8} \frac{\Delta \rho g r^{4}}{\mu} \sum \phi_{i} \int_{0}^{F_{i(\max )}} w d F_{i}
$$

which is modified from the equation for volume flux through a deformable conduit [Turcotte and Schubert, 2002]. $F_{i(\max )}$ is the melt fraction of lithology $i$ at the top of the conduit, $r$ is the conduit radius (default: $100 \mathrm{~km}$ ), and $\mu$ is the viscosity of the plume (default: $10^{19} \mathrm{Pas}$ ), with the default values taken from Shorttle et al. [2014].

If the meltingColumn class used to initialise the intraPlate class has chemistry and the melt compositions represent instantaneous melts, they will be homogenised during initialisation according to:

$$
\bar{C}=\frac{\sum \phi_{i} \int_{0}^{F_{i(\max )}} w C_{i} d F_{i}}{\sum \phi_{i} \int_{0}^{F_{i(\max )}} w d F_{i}}
$$

If the melt compositions represent accumulated melts and there is a weighting function applied, no result will be returned.

\section{Acknowledgements}

This work was supported by Natural Environment Research Council studentship [NE/L002574/1] awarded to KW.

\section{Author CONTRIBUtions}

SM conceived the idea of a modular mantle melting engine built in Python, and designed the over- 
arching structure of the library. SM and KW wrote the code for the melting models, developed the documentation, and drafted the manuscript. KW led the development of the INVMEL chemistry code and many of the features in the chemistry module. MG developed the hydrous melting code, which was generalised by SM. All authors contributed to editing the manuscript.

\section{Data AVAilability}

The latest version of pyMelt can be obtained from github.com/simonwmatthews/pyMelt and the pip package manager. pyMelt version v1.960 is archived in a Zenodo repository with DOI $10.5281 /$ zenodo.6013925.

\section{REFERENCES}

Asimow, P. D., Langmuir, and $\mathrm{CH}$ (2003). The importance of water to oceanic mantle melting regimes. Nature, 421(6925):815-820.

Ball, P., Duvernay, T., and Davies, D. R. (2022). A coupled geochemical-geodynamical approach for predicting mantle melting in space and time. Geochemistry, Geophysics, Geosystems, in press.

Ball, P., White, N., Maclennan, J., and Stephenson, S. (2021). Global influence of mantle temperature and plate thickness on intraplate volcanism. $\mathrm{Na}$ ture communications, 12(1):1-13.

Brown, E. L. and Lesher, C. E. (2014). North Atlantic magmatism controlled by temperature, mantle composition and buoyancy. Nature Geoscience, $7(11): 820-824$.

Brown, E. L. and Lesher, C. E. (2016). REEBOX PRO: A forward model simulating melting of thermally and lithologically variable upwelling mantle. Geochemistry, Geophysics, Geosystems, 17(10):3929-3968.

Gibson, S. and Geist, D. (2010). Geochemical and geophysical estimates of lithospheric thickness variation beneath Galápagos. Earth and Planetary Science Letters, 300(3-4):275-286.

Gleeson, M., Soderman, C., Matthews, S., Cottaar, S., and Gibson, S. (2021). Geochemical constraints on the structure of the Earth's deep mantle and the origin of the LLSVPs. Geochemistry, Geophysics, Geosystems, 22(9):e2021GC009932.

Jennings, E. S., Holland, T. J., Shorttle, O., Maclennan, J., and Gibson, S. A. (2016). The composition of melts from a heterogeneous mantle and the origin of ferropicrite: application of a thermodynamic model. Journal of Petrology, 57(1112):2289-2310.
Katz, R. F., Spiegelman, M., and Langmuir, C. H. (2003). A new parameterization of hydrous mantle melting. Geochemistry, Geophysics, Geosystems, $4(9)$.

Lambart, S., Baker, M. B., and Stolper, E. M. (2016). The role of pyroxenite in basalt genesis: Melt-PX, a melting parameterization for mantle pyroxenites between 0.9 and 5 GPa. Journal of Geophysical Research: Solid Earth, 121(8):5708-5735.

Maclennan, J., McKenzie, D., and Gronvöld, K. (2001). Plume-driven upwelling under central Iceland. Earth and Planetary Science Letters, 194(12):67-82.

Matthews, S., Shorttle, O., and Maclennan, J. (2016). The temperature of the Icelandic mantle from olivine-spinel aluminum exchange thermometry. Geochemistry, Geophysics, Geosystems, 17(11):4725-4752.

Matthews, S., Wong, K., Shorttle, O., Edmonds, M., and Maclennan, J. (2021). Do olivine crystallization temperatures faithfully record mantle temperature variability? Geochemistry, Geophysics, Geosystems, 22(4):e2020GC009157.

McKenzie, D. and Bickle, M. (1988). The volume and composition of melt generated by extension of the lithosphere. Journal of petrology, 29(3):625679.

McKenzie, D. and O'Nions, R. K. (1991). Partial melt distributions from inversion of rare earth element concentrations. Journal of Petrology, 32(5):10211091.

Pertermann, M. and Hirschmann, M. M. (2003). Partial melting experiments on a MORB-like pyroxenite between 2 and $3 \mathrm{GPa}$ : Constraints on the presence of pyroxenite in basalt source regions from solidus location and melting rate. Journal of Geophysical Research: Solid Earth, 108(B2).

Phipps Morgan, J. (2001). Thermodynamics of pressure release melting of a veined plum pudding mantle. Geochemistry, Geophysics, Geosystems, 2(4).

Plank, T. and Langmuir, C. H. (1992). Effects of the melting regime on the composition of the oceanic crust. Journal of Geophysical Research: Solid Earth, 97(B13):19749-19770.

Putirka, K. D. (2008). Thermometers and barometers for volcanic systems. Reviews in mineralogy and geochemistry, 69(1):61-120.

Shorttle, O., Maclennan, J., and Lambart, S. (2014). Quantifying lithological variability in the mantle. Earth and Planetary Science Letters, 395:24-40. 
Smith, P. M. and Asimow, P. D. (2005). Adiabat_1ph: A new public front-end to the melts, pmelts, and phmelts models. Geochemistry, Geophysics, Geosystems, 6(2).

Turcotte, D. L. and Schubert, G. (2002). Geodynamics. Cambridge university press.

Virtanen, P., Gommers, R., Oliphant, T. E., Haberland, M., Reddy, T., Cournapeau, D., Burovski, E., Peterson, P., Weckesser, W., Bright, J., van der Walt, S. J., Brett, M., Wilson, J., Millman, K. J., Mayorov, N., Nelson, A. R. J., Jones, E., Kern, R., Larson, E., Carey, C. J., Polat, İ., Feng, Y., Moore, E. W., VanderPlas, J., Laxalde, D., Perktold, J., Cimrman, R., Henriksen, I., Quintero, E. A., Harris, C. R., Archibald, A. M., Ribeiro, A. H., Pedregosa, F., van Mulbregt, P., and SciPy 1.0 Contributors (2020). SciPy 1.0: Fundamental Algorithms for Scientific Computing in Python. Nature Methods, 17:261-272.

White, R. S., McKenzie, D., and O'Nions, R. K. (1992). Oceanic crustal thickness from seismic measurements and rare earth element inversions. Journal of Geophysical Research: Solid Earth, 97(B13):19683-19715. 